\title{
Green tea (Camellia sinensis) and cancer prevention: a systematic review of randomized trials and epidemiological studies Jianping Liu*1,2,3, Jianmin Xing ${ }^{1}$ and Yutong Fei ${ }^{1}$
}

\author{
Address: ${ }^{1}$ Centre for Evidence-Based Chinese Medicine, Beijing University of Chinese Medicine, Beijing 100029, PR China, ${ }^{2}$ National Research \\ Centre in Complementary and Alternative Medicine (NAFKAM), University of Tromso, Norway and ${ }^{3}$ Division of Chinese Medicine, RMIT \\ University, Melbourne, Australia \\ Email: Jianping Liu* - Jianping.Liu@fagmed.uit.no; Jianmin Xing - xjm761002@163.com; Yutong Fei - yutong_fei@yahoo.com.cn \\ * Corresponding author
}

Published: 22 October 2008

Chinese Medicine 2008, 3:12 doi:10.1186/1749-8546-3-12

This article is available from: http://www.cmjournal.org/content/3/I/I2

(C) 2008 Liu et al; licensee BioMed Central Ltd.

This is an Open Access article distributed under the terms of the Creative Commons Attribution License (http://creativecommons.org/licenses/by/2.0), which permits unrestricted use, distribution, and reproduction in any medium, provided the original work is properly cited.
Received: 23 May 2008

Accepted: 22 October 2008

\begin{abstract}
Background: Green tea is one of the most popular beverages worldwide. This review summarizes the beneficial effects of green tea on cancer prevention.

Methods: Electronic databases, including PubMed (1966-2008), the Cochrane Library (Issue I, 2008) and Chinese Biomedical Database (1978-2008) with supplement of relevant websites, were searched. There was no language restriction. The searches ended at March 2008. We included randomized and non-randomized clinical trials, epidemiological studies (cohort and case-control) and a meta-analysis. We excluded case series, case reports, in vitro and animal studies. Outcomes were measured with estimation of relative risk, hazard or odd ratios, with $95 \%$ confidence interval.

Results: Forty-three epidemiological studies, four randomized trials and one meta-analysis were identified. The overall quality of these studies was evaluated as good or moderate. While some evidence suggests that green tea has beneficial effects on gastrointestinal cancers, the findings are not consistent.
\end{abstract}

Conclusion: Green tea may have beneficial effects on cancer prevention. Further studies such as large and long term cohort studies and clinical trials are warranted.

\section{Background}

Tea is one of the most popular beverages around the world. Popular in countries such as China and Japan, green tea accounts for $20 \%$ of tea consumption worldwide [1].

Green tea is derived from Camellia sinensis, an evergreen shrub of the Theaceae family. Unlike black tea which is fermented, green tea is produced in a non-fermented process. Green tea may be consumed in the form of a brewed beverage or capsular extract. In some countries, tea is used as dietary supplements. In China the medicinal use of green tea dated back to 4,700 years ago. Currently, there is no established recommended dose for green tea extract. Researchers examined the effects of habitually green tea drinking on cancer prevention; however, evidence has not been corroborated [2-4].

The main active ingredients of green tea include polyphenolic compounds such as epicatechin (EC), epicatechin-3gallate (ECG), epigallocatechin (EGC) and epigallocatechin-3-gallate (EGCG), all of which may be responsible for the anti-carcinogenic and anti-mutagenic activities of green tea. Other polyphenols in green tea include fla- 
vanols and their glycosides and depsides such as cholorogenic acid, quinic acids, carotenoids, trigalloylglucose, lignin, protein, chlorophyll, minerals (aluminum or manganese, depending on the soil content), caffeine and a very small amount of methylxanthines [5].

Polyphenols in green tea were shown to be powerful antioxidants with anti-carcinogenic properties [6]. Human studies on pharmacokinetics of polyphenols in green tea were conducted $[7,8]$. The evidence suggests that ingested polyphenols and their metabolites play a role in the action against gastrointestinal cancers.

Many in vitro and in vivo studies demonstrated that polyphenols from green tea were anti-carcinogenic by inducing apoptosis and inhibiting cell-growth, cyclindependent kinase inhibitor and urokinase (an enzyme crucial for cancer growth) [1]. Probable action mechanisms include antioxidant and free-radical scavenging activity and stimulation of detoxification systems through selective induction or modification of phase I and II metabolic enzymes.

In light of growing research findings on green tea, we conducted a systematic review of randomized clinical trials and epidemiological studies to summarize the current evidence of its beneficial and harmful effects on cancer prevention in humans.

\section{Methods}

\section{Databases and search strategy}

We searched various electronic databases, including PubMed (1966-2008) [9], the Cochrane Library (Issue 1, 2008; CD-ROM), Chinese Biomedical Database (CDROM, 1978-2008). No language restriction was applied. The searches ended at March 2008. The search terms used included cancer, neoplasm, green tea, tea, Camellia sinensis, diet therapy, case-control study, cohort study, clinical trial, review, systematic review and meta-analysis. We also searched the following websites to identify eligible studies: NCCAM [10], the Cancer Society [11], Complementary/Integrative Medicine Education Resources (CIMER) [12], NCI Cancer Information Summaries: Complementary and Alternative Medicine [13] and the Memorial Sloan-Kettering Cancer Center [14].

\section{Quality assessment}

As various study designs (i.e. randomized, non-randomized, prospective cohort and case-control) are included in this review, categorization after quality assessment was applied to each study [15] as follows.

\section{Category A (good)}

Studies in category A have the least biases and their results are considered valid. These studies are likely to consist of
(1) clear description of the population, setting, interventions and comparison groups; (2) appropriate measurement of outcomes; (3) appropriate statistical and analytical methods; (4) no reporting errors; (5) less than 20 percent dropouts; (6) clear reporting of dropouts; and (7) appropriate consideration and adjustment for potential confounders.

\section{Category B (fair)}

Studies in category B are susceptible to some degrees of biases that are not sufficient to invalidate the results. These studies may have sub-optimal adjustments for potential confounders and may also lack certain information that is needed to assess limitations and potential problems.

\section{Category $C$ (poor)}

Studies in category $\mathrm{C}$ have significant biases which may invalidate the results. These studies either do not consider potential confounders or do not make adjustments for them appropriately. These studies may have critical flaw in design, analysis and/or reporting, missing information and/or discrepancies in reporting.

\section{Inclusion criteria}

We included randomized controlled trials, controlled clinical trials or observational studies including prospective cohort and case-control studies on cancer prevention of green tea oral consumption in healthy individuals or cancer patients. Systematic reviews or meta-analyses were also included. In this review, only studies in the above mentioned categories A and B were included.

\section{Exclusion criteria}

We excluded case series, case reports, in vitro cell culture and animal studies. Poor quality studies (i.e. those in Category C) were also excluded.

\section{Data abstraction and analysis}

Relative risk (RR) or hazard ratio (HR) was used for clinical trials or cohort studies, while odds ratio (OR) was used for case-control studies. The above effect estimation was presented with 95\% confidence interval (95\% CI).

Due to the heterogeneity of study designs, settings, interventions and outcomes, we did not conduct a meta-analysis.

\section{Results}

A Cochrane systematic review on green tea for cancer (protocol stage) published in 2004 does not include any studies [16]. This review identified 48 clinical studies investigating the consumption of green tea and its association with the risk of developing cancer, including 42 epidemiological studies [17-58], one phase I trial [59], four 
randomized trials [60-63] and one meta-analysis [64] (Additional file 1). Twenty-two and 20 of the 42 epidemiological studies were cohort studies and case-control studies respectively, among which two studies [26,43] reported data from four cohort studies and one cohort study $[17,18]$ and one case-control study $[41,42]$ were reported twice. All studies were published between 1984 and 2008; 44 studies were published in international journals, three in Chinese $[28,32,56]$ and one in Japanese [38].

Participants in the included studies ranged from healthy individuals, pre-cancer patients to cancer patients. Several studies reported various cancers in their cases or cohorts, but in this review these data were presented separately in relevant categories. Average sample size for cohort studies was 31,798 (from 52 to 102,137 per study), 1,099 for case-control studies (from 213 to 3,818 per study) and 182 for randomized trials (from 60 to 400 per trial). Decaffeinated green tea, tea polyphenols (500 mg or 1000 $\mathrm{mg}$ ) or catechins was used for 3-12 months in randomized trials, whereas regular drinking tea was used for a fixed period or lifelong time in epidemiological studies. The outcomes reported from randomized trials included 8-hydroxydeoxyguanosine (8-OHdG) in urine (an indicator for oxidative DNA damage) $[60,62]$, histopathological examination [61] and incidence of cancer [63]. Risk of cancer development was the main outcome for epidemiological studies; a few studies reported mortality $[20,23]$, recurrence $[47,49]$, survival of cancer [57] and sister chromatid exchange (SCE) rates [21].

Four randomized trials were placebo-controlled, doubleblinded (three) and of acceptable methodological quality in terms of randomization, blinding and reporting of the studies. According to the generic quality grading for all included studies, 29 studies $(60 \%)$ were evaluated as good (A) and the remaining as fair (B).

\section{Cancer prevention}

Caner in general (5 studies)

A cohort study of 8,552 people with nine years of followup showed a negative association of green tea consumption with cancer incidence, especially among Japanese women drinking more than ten cups a day (RR 0.59; 95\% CI, 0.35-0.98) [17,18]. However, a larger cohort study with 38,540 people in Japan did not show an association between green tea consumption and sum incidence of all cancers (RR 1.0; 95\% CI, 0.91-1.1 for those drinking two to four times per day; RR 0.98; 95\%CI, $0.88-1.1$ for those drinking five times or more per day, both compared with those drinking one time or less per day) [19]. Another large cohort study found no significant benefit in terms of cancer mortality among a total of 1,134 cancer patients who consumed green tea and those who did not [20]. A randomized trial comparing green tea, black tea with water in 143 heavy smokers found significant decrease in 8-OHdG levels after a 4-month intervention [60]. A prospective cohort study in 52 male smokers demonstrated that drinking green tea inhibited cigarette-induced increase in sister chromatid exchange rates [21].

\section{Oral and esophageal cancer (8 studies)}

A prospective cohort study followed 20,550 men and 29,671 women for an average of 10.3 years and estimated the HRs $(95 \% \mathrm{CI})$ in oral cancer [22]. For women, the HRs $(95 \% \mathrm{CI})$ were $0.51(0.10-2.68), 0.60(0.17-2.10)$ and $0.31(0.09-1.07)$ for green tea consumption of one to two, three to four and five or more cups per day respectively, compared with those who drank less than one cup per day ( $P$ for trend, 0.08). For men, no trend was observed.

Inconsistent findings exist in case-control and cohort studies on green tea drinking and esophageal cancer [2328]. Two population-based case-control studies of 3,049 subjects found a protective effect of green tea drinking against esophageal cancer among women $[24,25]$, while another case-control study of 1,043 subjects showed 39\% decrease of risk of esophageal cancer among alcoholic drinkers and 31\% decrease among smokers [28]. The tea polyphenol epigallocatechin in urine was inversely associated with cancer risk when the data of gastric and esophageal cancer sub-sites were combined [27], indicating protective effect of green tea. However, in terms of death, a retrospective cohort and a pooled analysis of two cohort studies with a total of 26,723 subjects demonstrated a positive association (HR 1.67; 95\% CI, 0.89-3.16) ( $P$ for trend, 0.04 ) of drinking green tea and mortality of esophageal cancer among men $[23,26]$. A randomized controlled trial with 400 participants who were pathologically confirmed to have esophageal precancerous lesions did not show benefit of decaffeinated green tea for alleviating precancerous lesions or abnormal cell proliferation compared with calcium as placebo [61].

\section{Stomach cancer (I I studies)}

Seven epidemiological studies (one cohort and six casecontrol studies) with a total number of 77,777 subjects showed inverse association of green tea consumption (urine polyphenol epigallocatechin in one study) and the risk reduction of stomach cancer [27,28,30,32,34,36,37]. The cohort study with 72,943 subjects showed benefit for women who consumed five or more cups of green tea per day (RR $0.51 ; 95 \% \mathrm{CI}, 0.30-0.86$ ) compared with one cup per day [30]. However, four studies including two cohort studies with 102,179 subjects did not show an inverse association of green tea consumption and risk reduction of stomach cancer or cancer-caused death $[29,30,33,35]$. 


\section{Pancreatic cancer (4 studies)}

Two case-control studies (in three publications) with 522 pancreatic cancer patients and 1,694 controls showed an inverse association of drinking green tea and the risk of pancreatic cancer [38,41,42]. By contrast, a hospital-based case-control study of 124 patients and 124 controls demonstrated a positive association of drinking five cups or more green tea per day and pancreatic cancer [39]. A population-based cohort study involving 102,137 participants with 11 years of follow-up did not find any association of the risk of pancreatic cancer and drinking green tea [40].

\section{Liver cancer (2 studies)}

A population-based case-control study involving 204 patients and 415 controls reported that drinking green tea reduced the risk of liver cancer by $78 \%$ (OR 0.25; 95\% CI, $0.11-0.57$ ) among alcoholic drinkers and by $43 \%$ among smokers [28]. A randomized, double-blinded, placebocontrolled trial in 124 individuals with sero-positive HBsAg and aflatoxin-albumin adducts showed a significant decrease of 8-hydroxydeoxyguanosine after three months of green tea polyphenols intake [62].

\section{Biliary ducts cancer (I study)}

Statistical analysis showed that green tea consumption was positively associated with the mortality of biliary duct cancer in a retrospective cohort study $(P \leq 0.01)$ [23].

\section{Colorectal cancer (6 studies)}

Two case-control studies (in three publications) involving 2,036 patients with colorectal cancer and 2,130 controls found that drinking green tea reduced the risk of colorectal cancer $[41,42,44]$. Gender difference was observed in two cohort studies $[45,46]$. A prospective cohort study that followed over 60,000 subjects for an average of 8.9 years found no statistically significant difference between green tea drinkers and non-drinkers (RR 1.12; 95\% CI, $0.97-1.29$ ) [45]. Another cohort study with six years of follow-up on 69,710 women found significant doseresponse relationship ( $\mathrm{RR} 0.63 ; 95 \% \mathrm{CI}, 0.45-0.88$ ) between regular and non-regular green tea drinkers [46]. By contrast, a pooled analysis from two cohort studies on over 60,000 subjects showed no association between drinking green tea and a lower risk of colorectal cancer [43].

\section{Breast cancer (5 studies)}

In a meta-analysis of two prospective cohorts of 35,004 Japanese women [64], green tea intake was not associated with a lower risk of breast cancer (222 cases); and the multivariate RR for women drinking more than five cups of green tea was 0.84 (95\% CI, $-0.57-1.24 ; P=0.69)$ compared with those drinking less than one cup per day. One case-control study showed significantly reduced risk of breast cancer by regular drinking a large amount of green tea [50]. However, a cohort study did not find an association of green tea intake with lower risk of breast cancer [48]. Another two cohort studies showed reduced recurrence of breast cancer among patients at stage I and II with high consumption of green tea (more than three cups per day) $[47,49]$.

\section{Lung cancer (4 studies)}

A randomized trial compared green tea and black tea with water in 143 heavy smokers for 4 months [60]. The content of 8-OHdG in urine was reduced among the subjects in green tea group, but not in black tea or water groups. A cohort study of 52 male adults, found that green tea drinking blocked cigarette-induced increase in sister chromatid exchange rates, suggesting potential protection against lung cancer [21]. Consumption of green tea was found to be associated with a reduced risk of lung cancer among non-smoking women in one case-control study [51]. A phase I dose finding study showed the maximum tolerated dose of green tea extract as $3 \mathrm{~g} / \mathrm{m}^{2}$ per day in patients with advanced lung cancer. The dose-limiting toxicities were diarrhea, nausea and hypertension [59].

\section{Prostate cancer (4 studies)}

A double-blind, placebo-controlled trial testing green tea catechins (600 mg per day for one year) significantly reduced the incidence of prostate cancer in a group of 60 volunteers with high-grade prostate intraepithelial neoplasia; no significant adverse effect was reported [63]. A case-control study found prostate cancer risk declined with increasing frequency, duration and quantity of green tea consumption [53]. A cohort study in 19,561 Japanese men showed that green tea intake was not associated with a lower risk of prostate cancer (HR 0.85; 95\% CI, 0.501.43 ) between men drinking five or more cups and less than one cup per day [54]. However, another recent cohort study with a larger sample size $(n=49,920)$ in Japan suggested that green tea was associated with a decreased risk of advanced prostate cancer (RR 0.52; 95\% CI, 0.28-0.96) in men drinking five or more cups compared with those drinking less than one cup per day [55].

\section{Urinary bladder cancer (I study and I ongoing trial)}

The risk of urinary bladder cancer was significantly reduced in women who consumed matcha (a powdered green tea) in a case-control study $(n=882)$ [52]. A phase II randomized, double-blind, placebo-controlled, multicenter trial $(n=330)$ is currently carried out in the United States [65].

\section{Endometrial cancer (I study)}

A population-based case-control study $(n=2082)$ suggested that regular green tea drinking reduced the risk of 
endometrial cancer (OR 0.74; 95\% CI, 0.54-1.01) in premenopausal women [56].

\section{Ovarian cancer (I study)}

A cohort study $(n=254)$ suggested that increasing the post-diagnosis consumption of green tea may boost the survival of patients of epithelial ovarian cancer [57].

\section{Adult leukemia (I study)}

A case-control study $(n=217)$ demonstrated statistical association (OR $0.51 ; 95 \%$ CI $0.27-0.96$ ) between higher intake of green tea and reduced risk of adult leukemia in a dose-response manner [58].

\section{Safety of green tea}

Green tea, as a popularly consumed beverage, is relatively non-toxic [66]. Phase I trial in 17 patients with advanced lung cancer showed that the maximum tolerated dose of green tea extract was $3 \mathrm{~g} / \mathrm{m}^{2}$ per day [59]. No severe adverse effects have been reported in association with the medicinal use of green tea [67]. Consumption of high doses of green tea or green tea extract (i.e. 5-6 litters per day) may cause nausea, vomiting, abdominal bloating/ pain, dyspepsia, flatulence and diarrhea [59,67]. Excessive consumption of caffeine from green tea may also cause central nervous system stimulation such as dizziness, insomnia, tremors, restlessness, confusion, diuresis (i.e. increasing urine output), heart rate irregularities and psychomotor agitation [67].

Human studies did not show severe adverse effects among volunteers who took 15 tablets of green tea per day (i.e. 2.25 g green tea extracts, 337.5 mg EGCG and 135 mg caffeine) for 6 months $[68,69]$. A randomized, placebo-controlled trial $(n=40)$ found no adverse effect in healthy individuals who took green tea polyphenols in the amount equivalent to the EGCG content in 8-16 cups of green tea once a day or twice a day in divided doses for four weeks [69].

\section{Discussion}

Current research findings are not sufficient to validate the effects of green tea on cancer prevention as most evidence coming from cohort (grade III) and case-control studies (grade IV) is not consistent. As tea drinking is common in many populations, it is difficult to randomize subjects into drinking groups or non-drinking groups. For the same reason, epidemiological studies are still important in this field.

This review of randomized trials and epidemiological studies shows the current evidence in green tea and cancer prevention. More than half of the studies (58\%) suggest that long-term consumption of green tea may reduce the risk of certain types of cancer, in particular gastrointestinal cancers, such as esophageal, stomach, pancreatic, liver and colorectal cancer. Stratified analysis suggests that women benefit more than men from green tea drinking. However, the beneficial effects are not consistent across all studies. The interpretation of these findings is a challenge due to the significantly heterogeneous study designs, settings, populations, exposures, comparisons, outcome measures and potential publication biases. The heterogeneity hinders meaningful meta-analysis despite the large number of studies covered in this systematic review. The discrepancies in the findings may be due to the following factors: (1) participants in terms of health status, family history of cancer, age, gender, ethnic and other lifestyle confounders such as smoking or alcohol drinking; (2) definitions of green tea consumption, e.g. frequency, duration, quantity of green tea and the quality of green tea products; and (3) study designs and trial settings.

This review shows that the overall evidence for protective effects of green tea against cancer is inconclusive. Therefore, further prospective cohort studies and clinical trials are warranted. Adequate sample size, better descriptions of populations and/or clear definitions of green tea consumption may be required for conclusive studies.

Moderate consumption of green tea (3-9 cups per day) is generally safe. People with known allergy/hypersensitivity to caffeine or tannin should avoid green tea. In general, the stimulatory effect of green tea is considerably less than that of coffee [66]. However, pregnant women, nursing mothers and patients with cardiac conditions are advised to avoid or limit their intake of green tea to two cups per day [68].

\section{Conclusion}

Great efforts have been made to show the beneficial effects of green tea consumption on various cancers. Some epidemiological studies demonstrated protective effects of green tea consumption on gastrointestinal, breast, lung and prostate cancer. However, these findings have not been confirmed by other studies covered in this review. Future prospective studies are therefore warranted.

\section{Competing interests}

The authors declare that they have no competing interests.

\section{Authors' contributions}

JPL conceived and drafted this article. JMX and YTF helped select studies and abstracted data. JPL validated the process and conducted data analysis. All authors contributed to the writing and approved the final version of the manuscript. 


\section{Additional material}

\section{Additional file 1}

Summary of the included studies on green tea for cancer prevention. The table provides summarized information of the studies included in the present systematic review, including names of authors, location, study design, study quality, type of cancer, population and main findings. Click here for file

[http://www.biomedcentral.com/content/supplementary/17498546-3-12-S1.doc]

\section{Acknowledgements}

JPL, JMX and YTF were supported by a grant from the National Basic Research Program of China ('973' Program, No.2006CB504602) and by the 'I I I' Project (B08006). JPL was also supported in part by a grant (No.R24 AT00 I 293) from the National Center for Complementary and Alternative Medicine (NCCAM) of the United States National Institutes of Health (NIH).

\section{References}

I. Green tea detailed scientific review [http://www.mdander son.org/departments/cimer/]

2. Brown MD: Green tea (Camellia sinensis) extract and its possible role in the prevention of cancer. Altern Med Rev 1999, 4:360-370.

3. Nakachi K, Matsuyama S, Miyake S, Suganuma M, Imai K: Preventive effects of drinking green tea on cancer and cardiovascular disease: epidemiological evidence for multiple targeting prevention. Biofactors 2000, 13:49-54.

4. Béliveau R, Gingras D: Green tea: prevention and treatment of cancer by nutraceuticals. Lancet 2004, 364: $1021-1022$

5. Yang CS, Chen L, Lee MJ, Balentine D, Kuo MC, Schantz SP: Blood and urine levels of tea catechins after ingestion of different amounts of green tea by human volunteers. Cancer Epidemiol Biomarkers Prev 1998, 7:35|-354.

6. Lambert JD, Yang CS: Mechanisms of cancer prevention by tea constituents. INutr 2003, 133:3262S-3267S.

7. Lee MJ, Maliakal P, Chen L, Meng X, Bondoc FY, Prabhu S, Lambert G, Mohr S, Yang CS: Pharmacokinetics of tea catechins after ingestion of green tea and (-)-epigallocatechin-3-gallate by humans: formation of different metabolites and individual variability. Cancer Epidemiol Biomarkers Prev 2002, I I:1025-1032.

8. Chow HH, Cai Y, Hakim IA, Crowell JA, Shahi F, Brooks CA, Dorr RT, Hara Y, Alberts DS: Pharmacokinetics and safety of green tea polyphenols after multiple-dose administration of epigallocatechin gallate and polyphenon $E$ in healthy individuals. Clin Cancer Res 2003, 9:3312-3319.

9. PubMed [http://www.ncbi.nlm.nih.gov/entrez/query.fcgi]

10. NCCAM [http://nccam.nih.gov/]

II. American Cancer Society [http://www.cancer.org/docroot/ home/index.asp]

12. Complementary/Integrative Medicine Education Resources (CIMER) [http://www.mdanderson.org/departments/CIMER/]

13. NCl Cancer Information Summaries: Complementary and Alternative Medicine [http://www.cancer.gov/cancerinfo/pdq/ $\mathrm{cam} /]$

14. Memorial Sloan-Kettering Cancer Center [http:// www.mskcc.org/mskcc/html// I570.cfm]

15. Agency for Healthcare Research and Quality [http:// www.ahrq.gov/downloads/pub/evidence/pdf/brfout/brfout.pdf]

16. Schmidt K, Horneber M, Borrelli F, Ernst E: Green tea (Camellia sinensis) for the prevention of cancer. (Protocol). Cochrane Database of Systematic Reviews 2004.

17. Imai K, Suga K, Nakachi K: Cancer-preventive effects of drinking green tea among a Japanese population. Prev Med 1997, 26:769-775.

18. Nakachi K, Matsuyama S, Miyake S, Suganuma M, Imai K: Preventive effects of drinking green tea on cancer and cardiovascular disease: epidemiological evidence for multiple targeting prevention. Biofactors 2000, 13:49-54.

19. Nagano J, Kono S, Preston DL, Mabuchi K: A prospective study of green tea consumption and cancer incidence, Hiroshima and Nagasaki (Japan). Cancer Causes Control 200 I, I 2:50 I-508.

20. Kuriyama S, Shimazu T, Ohmori K, Kikuchi N, Nakaya N, Nishino Y, Tsubono Y, Tsuji I: Green tea consumption and mortality due to cardiovascular disease, cancer, and all causes in Japan: the Ohsaki study. JAMA 2006, 296: I255-I 265.

2I. Shim JS, Kang MH, Kim YH, Roh JK, Roberts C, Lee IP: Chemopreventive effect of green tea (Camellia sinensis) among cigarette smokers. Cancer Epidemiol Biomarkers Prev 1995, 4:387-391.

22. Ide R, Fujino Y, Hoshiyama Y, Mizoue T, Kubo T, Pham TM, Shirane K, Tokui N, Sakata K, Tamakoshi A, Yoshimura T, JACC Study Group: A prospective study of green tea consumption and oral cancer incidence in Japan. Ann Epidemiol 2007, 17:821-826.

23. Hara N, Sakata K, Nagai M, Fujita Y, Hashimoto T, Yanagawa H: Statistical analyses on the pattern of food consumption and digestive-tract cancers in Japan. Nutr Cancer 1984, 6:220-228.

24. Gao YT, McLaughlin JK, Blot WJ, Ji BT, Dai Q, Fraumeni JF Jr: Reduced risk of oesophageal cancer associated with green tea consumption. J Natl Cancer Inst 1994, 86:855-858.

25. Wang JM, Xu B, Rao JY, Shen HB, Xue HC, Jiang QW: Diet habits, alcohol drinking, tobacco smoking, green tea drinking, and the risk of esophageal squamous cell carcinoma in the Chinese population. Eur I Gastroenterol Hepatol 2007, 19: I 7I- I76.

26. Ishikawa $A$, Kuriyama $S$, Tsubono $Y$, Fukao A, Takahashi $H$, Tachiya $\mathrm{H}$, Tsuji I: Smoking, alcohol drinking, green tea consumption and the risk of esophageal cancer in Japanese men. J Epidemiol 2006, 16: 185-192.

27. Sun CL, Yuan JM, Lee MJ, Yang CS, Gao YT, Ross RK, Yu MC: Urinary tea polyphenols in relation to gastric and oesophageal cancers: a prospective study of men in Shanghai, China. Carcinogenesis 2002, 23: | 497-I503.

28. Mu LN, Zhou XF, Ding BG, Wang RH, Zhang ZF, Chen CW, Wei GR, Zhou XM, Jiang QW, Yu SZ: A case-control study on drinking green tea and decreasing risk of cancers in the alimentary canal among cigarette smokers and alcohol drinkers. Zhonghua Liu Xing Bing Xue Za Zhi 2003, 24(3): 192-195.

29. Hoshiyama Y, Kawaguchi T, Miura Y, Mizoue T, Tokui N, Yatsuya H Sakata K, Kondo T, Kikuchi S, Toyoshima H, Hayakawa N, Tamakoshi A, Ohno Y, Yoshimura T: A prospective study of stomach cancer death in relation to green tea consumption in Japan. $\mathrm{Br}$ J Cancer 2002, 87:309-3।3.

30. Sasazuki S, Inoue M, Hanaoka T, Yamamoto S, Sobue T, Tsugane S: Green tea consumption and subsequent risk of gastric cancer by subsite: the JPHC Study. Cancer Causes Control 2004, 15:483-491.

3I. Tsubono Y, Nishino Y, Komatsu S, Hsieh CC, Kanemura S, Tsuji I, Nakatsuka H, Fukao A, Satoh H, Hisamichi S: Green tea and the risk of gastric cancer in Japan. N Engl J Med 200I, 344:632-636.

32. Yu SZ, Zhang ZF, Yu GP, Zhu WD, Li YL, Wang MR, Guo CH, Xie $Z C$ : Epidemiological study of the influence of drinking green tea on gastric cancer and chronic gastritis incidence. Chin J Cancer 200I, I I:4I-45.

33. Hoshiyama $Y$, Kawaguchi T, Miura $Y$, Mizoue T, Tokui N, Yatsuya $\mathrm{H}$ Sakata K, Kondo T, Kikuchi S, Toyoshima H, Hayakawa N, Tamakoshi A, Ohno Y, Yoshimura T: A nested case-control study of stomach cancer in relation to green tea consumption in Japan. $\mathrm{Br}$ J Cancer 2004, 90:135-138.

34. Kono S, Ikeda M, Tokudome S, Kuratsune M: A case-control study of gastric cancer and diet in northern Kyushu, Japan. Jpn J Cancer Res 1988, 79:1067-1074.

35. Ji BT, Chow WH, Yang G, McLaughlin JK, Gao RN, Zheng W, Shu $X O$, Jin $F$, Fraumeni JF Jr, Gao YT: The influence of cigarette smoking, alcohol, and green tea consumption on the risk of carcinoma of the cardia and distal stomach in Shanghai, China. Cancer 1996, 77:2449-2457.

36. Yu GP, Hsieh CC, Wang LY, Yu SZ, Li XL, Jin TH: Green-tea consumption and risk of stomach cancer: a population-based case-control study in Shanghai, China. Cancer Causes Control 1995, 6:532-538.

37. Tajima K, Tominaga S: Dietary habits and gastro-intestinal cancers: a comparative case-control study of stomach and large intestinal cancers in Nagoya, Japan. Jpn I Cancer Res 1985, 76:705-716 
38. Goto R, Masuoka H, Yoshida K, Mori M, Miyake H: A case control study of cancer of the pancreas. Gan No Rinsho 1990, Spec No:344-350.

39. Mizuno S, Watanabe S, Nakamura K, Omata M, Oguchi H, Ohashi K, Ohyanagi H, Fujiki T, Motojima K: A multi-institute case-control study on the risk factors of developing pancreatic cancer. Jpn J Clin Oncol 1992, 22:286-29I.

40. Luo J, Inoue M, Iwasaki M, Sasazuki S, Otani T, Ye W, Tsugane S, JPHC Study Group: Green tea and coffee intake and risk of pancreatic cancer in a large-scale, population-based cohort study in Japan (JPHC study). Eur J Cancer Prev 2007, I 6:542-548.

4I. Ji BT, Chow WH, Hsing AW, McLaughlin JK, Dai Q, Gao YT, Blot WJ, Fraumeni JF Jr: Green tea consumption and the risk of pancreatic and colorectal cancers. Int J Cancer 1997, 70:255-258.

42. Dai Q, Ji BT, Jin F, Xu M, Gao YT: Tobacco, alcohol and green tea consumption and pancreatic cancer: a population-based case-control study in urban Shanghai, China. Cancer 1996, 16:5-10.

43. Suzuki Y, Tsubono Y, Nakaya N, Koizumi Y, Suzuki Y, Shibuya D, Tsuji I: Green tea and the risk of colorectal cancer: pooled analysis of two prospective studies in Japan. J Epidemiol 2005, I 5: I 18-124.

44. Kato I, Tominaga S, Matsuura A, Yoshii Y, Shirai M, Kobayashi S: A comparative case-control study of colorectal cancer and adenoma. JPn J Cancer Res 1990, 8 I: I I0I-II08.

45. Sun CL, Yuan JM, Koh WP, Lee HP, Yu MC: Green tea and black tea consumption in relation to colorectal cancer risk: the Singapore Chinese Health Study. Carcinogenesis 2007, 28:2|43-2|48.

46. Yang G, Shu XO, Li H, Chow WH, Ji BT, Zhang X, Gao YT, Zheng W: Prospective cohort study of green tea consumption and colorectal cancer risk in women. Cancer Epidemiol Biomarkers Prev 2007, 1 6: I219-1223.

47. Inoue M, Tajima K, Mizutani M, Iwata $H$, Iwase T, Miura S, Hirose $K$, Hamajima N, Tominaga S: Regular consumption of green tea and the risk of breast cancer recurrence: follow-up study from the Hospital-based Epidemiologic Research Program at Aichi Cancer Center (HERPACC), Japan. Cancer Lett 200I, 167:175-182.

48. Key TJ, Sharp GB, Appleby PN, Beral V, Goodman MT, Soda M, Mabuchi K: Soya foods and breast cancer risk: a prospective study in Hiroshima and Nagasaki, Japan. Br J Cancer 1999, 8 I: I248-1256.

49. Nakachi K, Suemasu K, Suga K, Takeo T, Imai K, Higashi Y: Influence of drinking green tea on breast cancer malignancy among Japanese patients. Jpn J Cancer Res I998, 89:254-26I.

50. Wu AH, Yu MC, Tseng CC, Hankin J, Pike MC: Green tea and risk of breast cancer in Asian Americans. Int J Cancer 2003, 1 06:574-579.

51. Zhong L, Goldberg MS, Gao YT, Hanley JA, Parent ME, Jin F: A population-based case-control study of lung cancer and green tea consumption among women living in Shanghai, China. Epidemiology 200I, I 2:695-700.

52. Ohno Y, Aoki K, Obata K, Morrison AS: Case-control study of urinary bladder cancer in metropolitan Nagoya. Natl Cancer Inst Monogr 1985, 69:229-234.

53. Jian L, Xie LP, Lee AH, Binns CW: Protective effect of green tea against prostate cancer: a case-control study in southeast China. Int J Cancer 2004, I 08: I 30- I 35.

54. Kikuchi N, Ohmori K, Shimazu T, Nakaya N, Kuriyama S, Nishino Y, Tsubono $Y$, Tsuji I: No association between green tea and prostate cancer risk in Japanese men: the Ohsaki Cohort Study. $\mathrm{Br} J$ Cancer 2006, 95:37I-373.

55. Kurahashi N, Sasazuki S, Iwasaki M, Inoue M, Tsugane S, JPHC Study Group: Green tea consumption and prostate cancer risk in Japanese men: a prospective study. Am J Epidemiol 2008, 167:71-77.

56. Gao J, Xiang YB, Xu WH, Shao CX, Ruan ZX, Cheng JR, Shu XO, Gao YT: Green tea consumption and the risk of endometrial cancer: a population-based case-control study in urban Shanghai. Zhonghua Liu Xing Bing Xue Za Zhi 2005, 26(5):323-327.

57. Zhang $M$, Lee $A H$, Binns $C W$, Xie $X$ : Green tea consumption enhances survival of epithelial ovarian cancer. Int J Cancer 2004, I | 2:465-469.
58. Zhang $M$, Zhao $X$, Zhang $X$, Holman $C D$ : Possible protective effect of green tea intake on risk of adult leukaemia. $\mathrm{Br} J \mathrm{Can}$ cer 2008, 98: 168-170.

59. Laurie SA, Miller VA, Grant SC, Kris MG, Ng KK: Phase I study of green tea extract in patients with advanced lung cancer. Cancer Chemother Pharmacol 2005, 55:33-38.

60. Hakim IA, Harris RB, Brown S, Chow HH, Wiseman S, Agarwal S, Talbot W: Effect of increased tea consumption on oxidative DNA damage among smokers: a randomized controlled study. I Nutr 2003, I 33:3303S-3309S.

61. Wang LD, Zhou Q, Feng CW, Liu B, Qi YJ, Zhang YR, Gao SS, Fan ZM, Zhou Y, Yang CS, Wei JP, Zheng S: Intervention and followup on human esophageal precancerous lesions in Henan, northern China, a high-incidence area for esophageal cancer. Gan to kagaku ryoho 2002, 29(suppl I): I59-I72.

62. Luo H, Tang L, Tang M, Billam M, Huang T, Yu J, Wei Z, Liang Y, Wang K, Zhang ZQ, Zhang L, Wang JS: Phase Ila chemoprevention trial of green tea polyphenols in high-risk individuals of liver cancer: modulation of urinary excretion of green tea polyphenols and 8-hydroxydeoxyguanosine. Carcinogenesis 2006, 27:262-268.

63. Bettuzzi S, Brausi M, Rizzi F, Castagnetti G, Peracchia G, Corti A: Chemoprevention of human prostate cancer by oral administration of green tea catechins in volunteers with high-grade prostate intraepithelial neoplassia: a preliminary report from a one-year proof-of-principle study. Cancer Res 2006, 66: $1234-1240$.

64. Suzuki Y, Tsubono Y, Nakaya N, Suzuki Y, Koizumi Y, Tsuji I: Green tea and the risk of breast cancer: pooled analysis of two prospective studies in Japan. Br J Cancer 2004, 90: I36 I- I 363.

65. National Cancer Institute: Erlotinib and green tea extract (Polyphenon ${ }^{\circledR} E$ ) in preventing cancer recurrence in former smokers who have undergone surgery for bladder cancer. [http://clinicaltrials.gov/ct/action/GetStudy]

66. Brown MD: Green tea (Camellia sinensis) extract and its possible role in the prevention of cancer. Altern Med Rev 1999 , 4:360-370.

67. The Natural Medicines Comprehensive Database [http:// www.naturaldatabase.com/]

68. Fujiki H, Suganuma M, Okabe S, Sueoka E, Suga K, Imai K, Nakachi K, Kimura S: Mechanistic findings of green tea as cancer preventive for humans. Proc Soc Exp Biol Med 1999, 220:225-228.

69. Chow HH, Cai Y, Hakim IA, Crowell JA, Shahi F, Brooks CA, Dorr RT, Hara Y, Alberts DS: Pharmacokinetics and safety of green tea polyphenols after multiple-dose administration of epigallocatechin gallate and polyphenon $E$ in healthy individuals. Clin Cancer Res 2003, 9:3312-3319.

Publish with Bio Med Central and every scientist can read your work free of charge

"BioMed Central will be the most significant development for disseminating the results of biomedical research in our lifetime. "

Sir Paul Nurse, Cancer Research UK

Your research papers will be:

- available free of charge to the entire biomedical community

- peer reviewed and published immediately upon acceptance

- cited in PubMed and archived on PubMed Central

- yours - you keep the copyright 